\title{
星状六(苯甲酸)的合成、晶体结构 及其铽配合物的发光性能*
}

\begin{abstract}
杨 迟 (1) (2)** 黄永德 ${ }^{(2)}$ 崔英德 ${ }^{\text {3 }}$ 陈小明 (2) 杨燕生 ${ }^{(4)}$
(1)广东工业大学轻工化工学院, 广州 510090; (2)香港大学化学系, 香港; (3)仲恺农业技术学院, 广州 510225;
\end{abstract} (4)中山大学化学系, 广州 510275)

\begin{abstract}
摘要通过简单的一步缩合反应合成了新型主体分子一一星状六苯甲酸 $\left(\mathbf{1 H}_{6}\right)$, 测定 了其 DMSO 加合物 $1 \mathrm{H}_{6} \cdot 6 \mathrm{DMSO}(\mathbf{2})$ 的晶体结构. 晶体属三方晶系, 空间群为 $\mathrm{R}-3$, 晶 胞参数 $a=b=2.4129(2), c=1.1576(3) \mathrm{nm}, \alpha=\beta=90^{\circ}, \gamma=120^{\circ}, V=5.837(2) \mathrm{nm}^{3}, Z=$ 3. 六个 DMSO 和氢键键接的端基苯甲酸交替排列在中心苯环的上下方, 形成“ $1,3,5$ 位基团向上；2，4，6-位基团向下”的双三棱柱结构. 在吡啶中制备了 1 的琙配合物 $\left(\mathrm{Tb}_{2} \mathrm{1}\right)_{2} \mathrm{Py} \cdot 9 \mathrm{H}_{2} \mathrm{O}(\mathbf{3})$, 该配合物发射明亮的铖离子特征荧光. 与相应苯甲酸琙的固体粉 末的荧光光谱相比, 化合物 $\mathbf{3}$ 有更高的发光强度和较长的激发态寿命. 说明 $\mathbf{1}$ 能很好地 包络中心琙离子, 是好的天线结构配体.
\end{abstract}

\section{关键词六取代苯 星状主体 硫醚羧酸 稀土 发光}

六取代苯类分子是一类重要的有机主体分子, 其六个取代基通常交替排列在中心苯环的 上下方, 形成一定大小的空穴, 可用来构筑选择包结特定客体分子的多孔结构的主体分子. 六 取代苯类主体分子的研究可以追溯到 20 世纪 70 年代 Vögtle 等人的开创性工作, 即基于六取 代苯的章鱼形分子(Octopus Molecules) ${ }^{[1]}$. 其后 MacNicol 等人进一步发展了这一体系, 他们模 拟酚类分子自组装的六聚体(Hexamer)笼状结构, 设计合成了系列新型的以刚性芳环为端基的 六取代苯类主体分子, 并依据其结构和功能特征命名为 “Hexa-host” ${ }^{\text {” }}$. 随后, 一系列六取代苯 类主体分子被设计合成出来，如 Toda 等的 “Hexa Pedals”[3], Foces-Foces 等的“Aromatic Propellenes" ${ }^{\text {" }}{ }^{[4]}$, 等等 ${ }^{[5 \sim 14]}$. 取代基的端基通常是具有一定刚性的芳环(Arms). 端基与中心苯环 的联结(Spacer)可以是刚性的双键、三键或芳香烃, 也可以是柔曲结构的脂肪链烃或醚键(或硫 醚键), 或直接与中心苯环键接. 为了利用分子间氢键或与金属配位形成扩展的一维、二维或 三维微孔结构超分子, 通常对端基进行修饰. 最主要的方法是用颈基 $(\mathrm{SH})^{[5]}$ 、氨基 $\left(\mathrm{NR}_{2}\right)^{[6 \sim 8]}$ 、 差基 $(\mathrm{OH})^{[9]}$ 、羧基 $(\mathrm{COOH})^{[10]}$ 、醛基 $(\mathrm{CHO})$ 或三键 ${ }^{[1]}$ 等对端基功能化. 功能基团的引人一般需 要多步反应, 通过保护-联结-去保护的途径完成.

功能六取代苯类分子可作为多臂配体与金属离子配位形成多种超分子微孔结构化合物, 但这方面的研究相对较少. Vögtle 和 Weber 最早研究了溶液中六取代苯类冠醚、链醚对部分金 属离子的萃取性能 ${ }^{[1]}$. Robson 等人报道了六咪唑取代苯与 $\mathrm{CdF}_{2}$ 配位形成的具有 $\alpha$-微孔的三维

2003-04-09 收稿, 2003-06-11 收修改稿

* 国家自然科学基金(批准号: 20105003)与广东省自然科学基金(批准号: 974174，990128)资助项目

** E-mail: yangchi@gdut.edu.cn 
网状结构 ${ }^{[12]}$. 其他如：Koten 等的六金属车轮分子 (Hexametallic cartwheel molecules) ${ }^{[13]}$. Schmidbaur 等人的金轮(Golden wheel)等 ${ }^{[14]}$. 我们曾报道了端基是氨基噻哒唑杂环的星状硫醚 HTT 与亚砜 HTS, 在酸性介质中分别选择包络一个和两个金属离子, 形成单核笼 $\left[\mathrm{M}(\mathrm{HTT}) \mathrm{Cl}_{2}\right]$ 和双核笼 $\left[\mathrm{M}_{2}(\mathrm{HTS}) \mathrm{Cl}_{4}\right]$, 研究了星状六胺 HTT、星状亚砜 HTS 及其铜包络物的电化学性质 ${ }^{[8]}$.

Kobayashi 等报道了刚性苯六(苯甲酸)(HCPB)的合成和晶体结构, HCPB 的功能端基苯甲 酸与中心苯环直接键接, 通过羧基间的氢键作用形成三角形微孔二维网状结构 ${ }^{[10]}$. 但端基与 中心苯环具有柔曲连接的苯六(苯甲酸)尚未见报道. 本文报道星状六(苯甲酸)(1)的合成、晶体 结构与功能性质. 1 的功能端基苯甲酸与中心苯环通过柔曲的硫醚键连接, 构象上有一定的流 变性. 主体分子 1 可通过简单的一步反应合成, 产率高, 产物易于纯化. 在有机碱存在下, 由 二甲亚砜中培养得到在空气中稳定的加合物 $1 \mathrm{H}_{6} \cdot 6 \mathrm{DMSO}(2)$, 测定了该加合物的晶体结构. 1 与铽离子作用形成 $\left(\mathrm{Tb}_{2} \mathbf{1}\right)_{2} \mathrm{Py} \cdot 9 \mathrm{H}_{2} \mathrm{O}(\mathbf{3})$ 配合物. 发光性能研究发现 3 比相应的苯甲酸铽配合物 有较长的激发态寿命和较高的发光效率.

\section{1 实验}

\section{1 仪器与试剂}

$\mathrm{C}, \mathrm{H}, \mathrm{N}$ 和 $\mathrm{S}$ 的元素分析在 Perkin-Elmer 240 自动测量仪上测定. 红外光谱用 Nicolet 170SX FT-IR 红外光谱仪测定(KBr 压片). 电子吸收光谱用 Shimadzu MPS-2000 紫外可见分光 光度计测定. ${ }^{1} \mathrm{H}$ 及 ${ }^{13} \mathrm{C}$ NMR 测定用 Bruker DPX-300 型波谱仪, TMS 作内标. 质谱(EI 和 FAB) 在 ZAB VG ANALY 质谱上测定.

硫代水杨酸、六甲基苯、 $\mathrm{Tb}\left(\mathrm{NO}_{3}\right)_{3} \cdot 6 \mathrm{H}_{2} \mathrm{O}$ 均为分析纯(ACROS). 金属钠、绝对乙醇、吡 啶及所有溶剂均为国产分析纯. 六溴甲基苯按文献方法由六甲基苯和过量溴素在 1,2-二溴乙 烷中回流溴化制得 ${ }^{[2]}$. 吡啶用前经蒸馏提纯.

\section{2 星状六(苯甲酸)的合成与表征}

在氮气保护下往 $330 \mathrm{~mL}$ 绝对乙醇中加人金属钠 $(6.9 \mathrm{~g}, 33 \mathrm{mmol})$ (注意: 氢气放出, 通风 䖞!), 待金属钠溶解(约需 $0.5 \sim 1.0 \mathrm{~h}$ ) 后, 将生成的无色透明的乙醇钠的乙醇溶液保持在 $60^{\circ} \mathrm{C}$. 搅拌和氮气氛下一次加人邻䘪基苯甲酸 $(4.7 \mathrm{~g}, 30 \mathrm{mmol})$, 在 $60^{\circ} \mathrm{C}$ 下继续搅拌 $0.5 \mathrm{~h}$, 得到邻颈 基苯甲酸二钠盐的白色悬浮液. 将所得的悬浮液冷至室温后加人六溴甲基苯 $(2.1 \mathrm{~g}, 3.3 \mathrm{mmol})$. 反应混合物在室温搅拌 $1 \mathrm{~h}$, 然后加热回流 $18 \mathrm{~h}$. 整个反应在氮气保护下进行. 反应完全后, 趁 热过滤, 滤饼用热乙醇充分洗涤. 将带浅黄色的滤饼溶于适量水中 $(300 \mathrm{~mL})$, 过滤除去不溶物. 滤液在冰浴冷却下用稀盐酸酸化至 $\mathrm{pH}=3$. 将白色沉淀过滤, 用水充分洗涤除去无机盐. 所得 滤饼用热乙醇充分抽提数次以除去未反应的邻巯基苯甲酸(至提取液呈无色). 所得白色固体 为 $1 \mathrm{H}_{6}$, 纯度可达到 $98 \%$ (HPLC), 产率 $92 \%\left(4.7 \mathrm{~g}\right.$ ), m.p. $309^{\circ} \mathrm{C}$ (分解). 分析样 $1 \mathrm{H}_{6} \cdot 6 \mathrm{DMSO}$ 由 二甲亚砜中重结晶得无色晶体. FAB MS: $m / z=1075 ;{ }^{1} \mathrm{H}$ NMR $\left(300 \mathrm{MHz},\left(\mathrm{CD}_{3}\right)_{2} \mathrm{SO}, 25^{\circ} \mathrm{C}\right.$, TMS): 4.31 (s, 2H, SCH2), $7.20\left(\mathrm{t}, J=7.2 \mathrm{~Hz}, 1 \mathrm{H}, \mathrm{Ph}-\mathrm{H}^{6}\right), 7.34$ (q, J = 7.6, 2H, Ph- $\left.\mathrm{H}^{4}, \mathrm{H}^{5}\right), 7.86$ $\left(\mathrm{d}, J=7.6,1 \mathrm{H}, \mathrm{Ph}-\mathrm{H}^{3}\right), 13.18(\mathrm{~s}, \mathrm{br}, 1 \mathrm{H}, \mathrm{COOH}) ;{ }^{13} \mathrm{C} \mathrm{NMR}\left(300 \mathrm{MHz},\left(\mathrm{CD}_{3}\right)_{2} \mathrm{SO}, 25^{\circ} \mathrm{C}, \mathrm{TMS}\right)$ : 124.06, 124.66, 126.52, 128.44, 130.67, 132.56, 135.65, 139.56, 167.53. IR (KBr): 1679, 1586, $1564,1465,1247,1148,1028,954,799,740,699,644 \mathrm{~cm}^{-1}$. 元素分析结果 $\left(\mathrm{C}_{66} \mathrm{H}_{78} \mathrm{O}_{18} \mathrm{~S}_{12}\right)$, 计算 值(\%): C51.34, H 5.09, S 24.92; 实验值(\%): C 51.39, H 5.10, S 25.08. 


\section{3 铽配合物 3 的合成与表征}

$1 \mathrm{H}_{6}(1 \mathrm{mmol})$ 加人到 $20 \mathrm{~mL}$ 重蒸馏过的吡啶中, 加热溶解得到无色透明的溶液. 搅拌下往 此热溶液中滴加 $\mathrm{Tb}\left(\mathrm{NO}_{3}\right)_{3} \cdot 6 \mathrm{H}_{2} \mathrm{O}$ 的甲醇溶液 $(0.05 \mathrm{~mol} / \mathrm{L}, 2.0 \mathrm{~mL})$, 立即析出白色沉淀. 将此 悬浮液继续加热搅拌 $5 \mathrm{~min}$, 室温放置沉化 $0.5 \mathrm{~h}$. 过滤, 用甲醇充分洗涤. 为了除去包结的溶 剂分子和结晶水, 在 $100{ }^{\circ} \mathrm{C}$ 下真空干燥过夜. 产物 $\left(\mathrm{Tb}_{2} \mathrm{1}\right)_{2} \mathrm{Py} \cdot 9 \mathrm{H}_{2} \mathrm{O}$ 为白色晶状粉末. 产率: $92 \%$. IR (KBr): 1589, 1572, 1537, 1463, 1436, 1404, 1060, 1043, 860, 745, 651, $489 \mathrm{~cm}^{-1}$. 元素分 析结果 $\left(\mathrm{Tb}_{4} \mathrm{C}_{113} \mathrm{H}_{95} \mathrm{NO}_{33} \mathrm{~S}_{12}\right)$, 计算值(\%): C 45.01, H 3.18, N 0.46, S 12.76; 实验值(\%): C 45.05, H 3.21, N 0.50, S 12.63.

\section{4 加合物 2 的晶体结构分析}

加合物 2 的晶体 X 射线衍射数据用 Rigaku AFC7R 型四圆衍射仪测定, $\operatorname{MoK} \alpha(\lambda=0.071073$ $\mathrm{nm}$ )射线、石墨单色器, $\omega-2 \theta$ 方式扫描. 以 24 个衍射点精确测定取向矩阵和晶胞参数, 所得数 据均经 $L p$ 因子校正和经验吸收校正. 晶体结构利用直接法解出, 非氢原子的位置由差值 Fourier 合成法获得. 结构由全对角最小二乘法优化, 除氢原子采用各向同性热参数修正外, 其 他原子均采用各向异性热参数修正, 最后的一致因子为 $R=0.054$ 和 $R_{w}=0.126$. 采用单位权 重, 最终差值 Fourier 图上的最大高度为 0.235. 晶体结构计算用 SHELXL93 程序包进行 ${ }^{[15]}$. 晶 体学数据及结构分析参数列于表 1 .

表 1 加合物 2 的晶体学数据及结构分析参数

\begin{tabular}{ll}
\hline 分子式 & $\mathrm{C}_{66} \mathrm{H}_{78} \mathrm{O}_{18} \mathrm{~S}_{12}$ \\
分子量 & 1544.00 \\
晶体大小 & $0.34 \mathrm{~mm} \times 0.28 \mathrm{~mm} \times 0.20 \mathrm{~mm}$ \\
实验温度 $/ \mathrm{K}$ & 294 \\
晶系 & 三方晶系 \\
空间群 & $\mathrm{R} \overline{3}$ \\
$a, b, c / \mathrm{nm}$ & $a=b=2.4129(2), c=1.1576(3)$ \\
$\alpha, \beta, \gamma /\left(^{\circ}\right)$ & $\alpha=\beta=90^{\circ}, \gamma=120^{\circ}$ \\
$V / \mathrm{nm}^{3}$ & $5.837(2)$ \\
$Z$ & 3 \\
$\rho_{\text {calc }} / \mathrm{mg}^{\circ} \cdot \mathrm{m}^{-3}$ & 1.318 \\
$F(000)$ & 2430 \\
$h, k, l$ & $-28 \leqslant h \leqslant 14,0 \leqslant k \leqslant 28,0 \leqslant l \leqslant 13$ \\
数据收集指数范围 $\left(\theta_{\text {max }}\right)$ & $25.0^{\circ}$ \\
总衍射点 & 2327 \\
独立衍射点 $(I>2.00 \sigma(I))$ & 2236 \\
$R, R_{w}[I>2 \sigma(I)]$ & $0.0547,0.1264$ \\
$R, R_{w}(\mathrm{all}$ data) & $0.0973,0.1515$ \\
$\mathrm{GOF}\left(F^{2}\right)$ & 1.023 \\
\hline
\end{tabular}

\section{2 结果与讨论}

\section{1 合成}

颈基水杨酸与乙醇钠在乙醇溶液中反应生成二钠盐, 然后与六溴甲基苯在回流搅拌下反 应 $18 \mathrm{~h}$, 得到目的产物 $1 \mathrm{H}_{6}$. 整个反应在惰性气氛下进行, 以避免颈基或硫醚键的氧化. 为了 避免羧酸钠与溴代烷的酯化副反应, 促进硫醚化的完成, 反应中采用了过量的巯基水杨酸(约 
为化学计量的 1.5 倍), 并采用先室温搅拌 $(0.5 \mathrm{~h}$ )后加热回流的措施. 反应完成后(TLC, 约 $18 \mathrm{~h}$ ), 趁热过滤,并用热乙醇充分洗涤.滤饼溶于适量水中, 过滤除去不溶物, 用稀盐酸酸化后析出粗 产物. 粗产物中尚含有少量未反应的颈基水杨酸, 用热乙醇洗涤除去. 产物 $1 \mathrm{H}_{6}$ 可用在 DMSO- $\mathrm{H}_{2} \mathrm{O}, \mathrm{DMF}-$ 二氧六环或吡定中重结晶的方法进一步提纯或生成溶剂加合物(式 1).

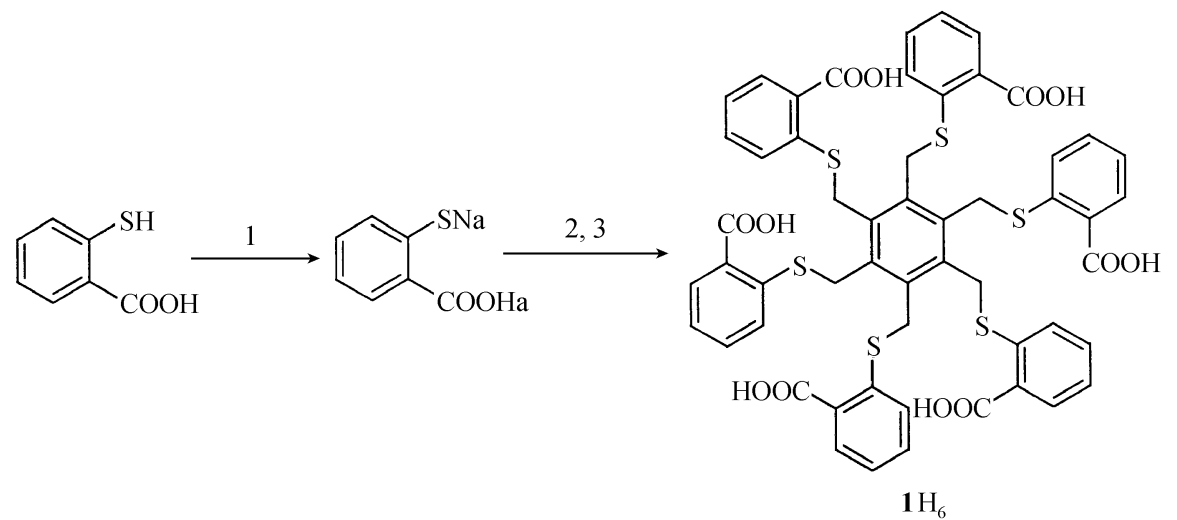

式 1

$\mathbf{1 H}_{6}$ 的合成图解. 1. EtONa, EtOH, $\mathrm{N}_{2} ; 2$. 六溴甲基苯, EtOH, $\mathrm{N}_{2}$, 回流 $18 \mathrm{~h} ; 3 . \mathrm{HCl}-\mathrm{H}_{2} \mathrm{O}$

我们发现, 溶剂加合物的形成与介质中存在的非包结类分子有关. $1 \mathrm{H}_{6}$ 溶于 DMSO, 首先 通过分子间氢键生成 $1 \mathrm{H}_{6} \cdot 6 \mathrm{DMSO}$ 缔合物, 该缔合物在结晶时可以包结溶剂分子或介质中存 在的其他小分子. 在 DMSO 中存在星状亚砜六胺主体分子 $\mathbf{H T S}^{[8]}$ 时, 可以得到在空气中稳定 的、不含包结其他溶剂小分子的加合物 $1 \mathrm{H}_{6} \cdot 6 \mathrm{DMSO}(2)$ 晶体. 由于 $\mathbf{2}$ 的单晶在空气中稳定, 可 直接用于常温下 $\mathrm{X}$ 射线单晶数据的收集. HTS 在 $1 \mathrm{H}_{6}$ 结晶中所起的作用可能与它的碱性有关.

六臂星状羧酸配体 $1 \mathrm{H}_{6}$ 的稀土配合物可用多种方法制备. $1 \mathrm{H}_{6}$ 与化学计量的氢氧化钠或氢 氧化钾的醇溶液作用得到 $1 \mathrm{Na}_{6}$ 或 $1 \mathrm{~K}_{6}$, 后者再与稀土离子(氯化物、硝酸盐或高氯酸盐等)作用 生成 $\mathbf{1}$ 的稀土配合物. $1 \mathrm{H}_{6}$ 与稀土离子的配位反应也可在有机碱(如吡啶、三乙胺、质子海绵等) 存在下进行. 在不同的条件下得到的稀土配合物的发光性能各异. 采用有机碱时, 产物中常含 有配位的有机碱. 由于 $1 \mathrm{H}_{6}$ 易溶于 $\mathrm{N}, \mathrm{N}$-二甲基甲酰胺和热吡啶, 因此, 在这两种碱性有机溶 剂中制备 $1 \mathrm{H}_{6}$ 的金属配合物最为合适. $\mathrm{DMF}$ 的碱性弱, $1 \mathrm{H}_{6}$ 与稀土盐的反应需要加热才能完成. 吡啶是较强的有机碱, 溶于吡啶中的六臂星状羧酸配体 $1 \mathrm{H}_{6}$ 与滴人的稀土离子反应迅速, 产物 以白色晶状粉末的形式沉淀出来, 经吡啶、甲醇洗涤后即得纯净产物.

\section{2 加合物 2 的晶体结构}

加合物的分子结构图示于图 1 和 2, 键长和键角数据见表 2 , 原子坐标见表 3 .

从分子结构看, 1 具有对称中心(图 1). 端基芳环与中心苯环基本垂直, 但不等同, 其夹角 分别是 86.41(34)，83.26(31) 和 81.75(34) ${ }^{\circ}$. 每个主体分子 1 与六个 DMSO 通过分子间氢键连 接. 形成氢键的羧基氢原子位于羧基外侧的氧原子上, 相应的氢键距离为 $\mathrm{O}(1) \mathrm{H} \cdots \mathrm{O}(3)=$ $0.2547(5) \mathrm{nm}$, 相关键角为 $\mathrm{S}(2)-\mathrm{O}(3) \ldots \mathrm{O}(1)=132.0(2) \mathrm{o}, \mathrm{C}(9)-\mathrm{O}(1) \ldots \mathrm{O}(3)=118.4(3)^{\circ}$. 六个 DMSO 和氢键键接的端基苯甲酸交替排列在中心苯环的上下方，形成“1，3，5-位基团向上；2, 4, 6-位基团向下”的构象，与酚类的六聚体的笼状结构一致. 


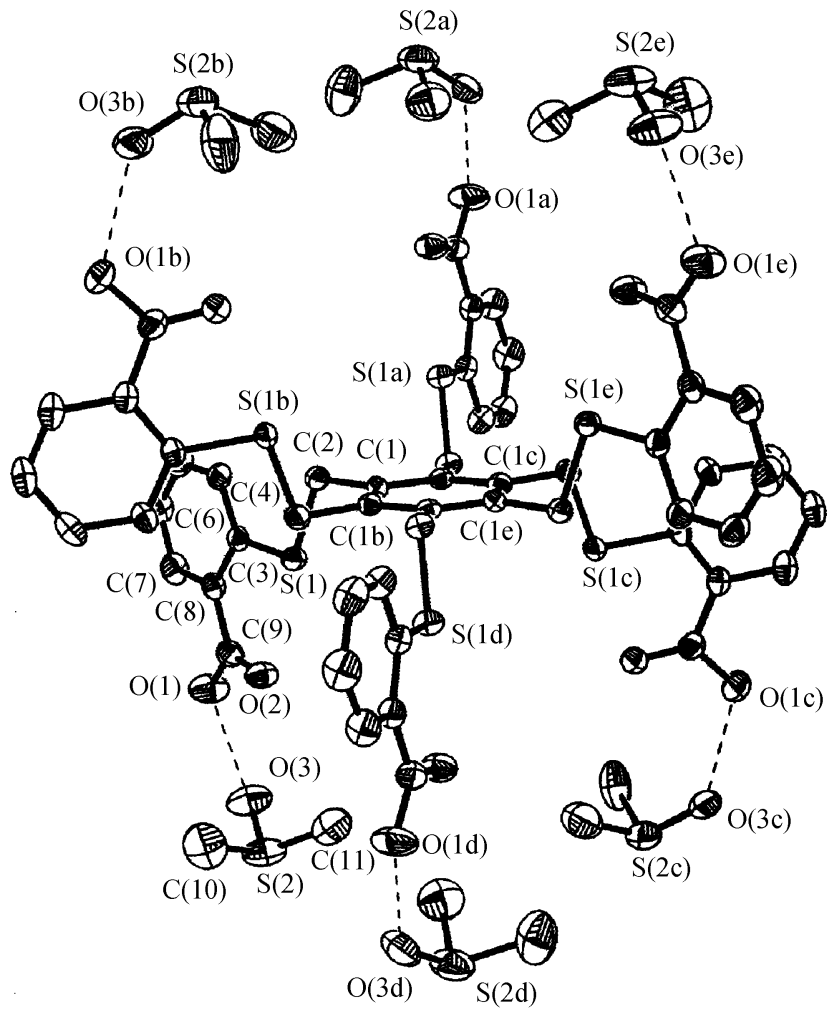

图 1 加合物 $\mathbf{2}$ 的 ORTEP 结构与原子编号示意图(虚线表示氢键)

表 22 的部分化学键长 $(\mathrm{nm})$ 、键角 $\left(^{\circ}\right)$

\begin{tabular}{ccc||cccc}
\hline 原子 & 原子 & 键长 & 原子 & 原子 & 原子 & 键角 \\
\hline $\mathrm{S}(1)$ & $\mathrm{C}(3)$ & $0.1774(4)$ & $\mathrm{C}(3)$ & $\mathrm{S}(1)$ & $\mathrm{C}(2)$ & $102.7(2)$ \\
$\mathrm{S}(1)$ & $\mathrm{C}(2)$ & $0.1829(4)$ & $\mathrm{C}(1) \# 1$ & $\mathrm{C}(1)$ & $\mathrm{C}(1) \# 2$ & $119.97(2)$ \\
$\mathrm{C}(1)$ & $\mathrm{C}(1) \# 1$ & $0.1399(3)$ & $\mathrm{C}(1) \# 1$ & $\mathrm{C}(1)$ & $\mathrm{C}(2)$ & $119.3(3)$ \\
$\mathrm{C}(1)$ & $\mathrm{C}(2)$ & $0.1508(5)$ & $\mathrm{C}(1) \# 2$ & $\mathrm{C}(1)$ & $\mathrm{C}(2)$ & $120.7(3)$ \\
$\mathrm{C}(3)$ & $\mathrm{C}(4)$ & $0.1389(5)$ & $\mathrm{C}(1)$ & $\mathrm{C}(2)$ & $\mathrm{S}(1)$ & $107.6(2)$ \\
$\mathrm{C}(3)$ & $\mathrm{C}(8)$ & $0.1404(5)$ & $\mathrm{C}(4)$ & $\mathrm{C}(3)$ & $\mathrm{C}(8)$ & $118.4(3)$ \\
$\mathrm{C}(4)$ & $\mathrm{C}(5)$ & $0.1386(5)$ & $\mathrm{C}(4)$ & $\mathrm{C}(3)$ & $\mathrm{S}(1)$ & $121.4(3)$ \\
$\mathrm{C}(5)$ & $\mathrm{C}(6)$ & $0.1364(6)$ & $\mathrm{C}(8)$ & $\mathrm{C}(3)$ & $\mathrm{S}(1)$ & $120.2(3)$ \\
$\mathrm{C}(6)$ & $\mathrm{C}(7)$ & $0.1367(6)$ & $\mathrm{C}(3)$ & $\mathrm{C}(4)$ & $\mathrm{C}(5)$ & $120.7(4)$ \\
$\mathrm{C}(7)$ & $\mathrm{C}(8)$ & $0.1396(5)$ & $\mathrm{C}(6)$ & $\mathrm{C}(5)$ & $\mathrm{C}(4)$ & $120.9(4)$ \\
$\mathrm{C}(8)$ & $\mathrm{C}(9)$ & $0.1478(6)$ & $\mathrm{C}(7)$ & $\mathrm{C}(6)$ & $\mathrm{C}(5)$ & $119.2(4)$ \\
$\mathrm{C}(9)$ & $\mathrm{O}(2)$ & $0.1207(5)$ & $\mathrm{C}(6)$ & $\mathrm{C}(7)$ & $\mathrm{C}(8)$ & $121.6(4)$ \\
$\mathrm{C}(9)$ & $\mathrm{O}(1)$ & $0.1313(5)$ & $\mathrm{C}(7)$ & $\mathrm{C}(8)$ & $\mathrm{C}(3)$ & $119.1(4)$ \\
$\mathrm{S}(2)$ & $\mathrm{O}(3)$ & $0.1488(4)$ & $\mathrm{C}(7)$ & $\mathrm{C}(8)$ & $\mathrm{C}(9)$ & $118.9(4)$ \\
$\mathrm{S}(2)$ & $\mathrm{C}(10)$ & $0.1727(7)$ & $\mathrm{O}(2)$ & $\mathrm{C}(9)$ & $\mathrm{O}(1)$ & $122.5(4)$ \\
$\mathrm{S}(2)$ & $\mathrm{C}(11)$ & $0.1749(6)$ & $\mathrm{O}(2)$ & $\mathrm{C}(9)$ & $\mathrm{C}(8)$ & $123.7(4)$ \\
\multicolumn{2}{c|}{} & & $\mathrm{O}(1)$ & $\mathrm{C}(9)$ & $\mathrm{C}(8)$ & $113.8(4)$ \\
\multicolumn{2}{|c|}{$\mathrm{O}(1) \ldots \mathrm{O}(3)$} & $\mathrm{O}(3)$ & $\mathrm{S}(2)$ & $\mathrm{C}(10)$ & $104.6(3)$ \\
$\mathrm{S}(2)-\mathrm{O}(3) \ldots \mathrm{O}(1)$ & $0.2547(5)$ & $\mathrm{O}(3)$ & $\mathrm{S}(2)$ & $\mathrm{C}(11)$ & $107.3(3)$ \\
$\mathrm{C}(9)-\mathrm{O}(1) \ldots \mathrm{O}(3)$ & $132.0(2)$ & $\mathrm{C}(10)$ & $\mathrm{S}(2)$ & $\mathrm{C}(11)$ & $98.1(3)$ \\
\hline
\end{tabular}


表 32 的原子坐标 $\left[\times 10^{4}\right]$ 和等效温度因子 $\left[\AA^{2} \times 10^{3}\right]$

\begin{tabular}{ccccc}
\hline 原子 & $x$ & $y$ & $z$ & $U_{i j}$ \\
\hline $\mathrm{S}(1)$ & $637(1)$ & $8971(1)$ & $8559(1)$ & $44(1)$ \\
$\mathrm{C}(1)$ & $232(2)$ & $9572(2)$ & $10010(3)$ & $35(1)$ \\
$\mathrm{C}(2)$ & $475(2)$ & $9105(2)$ & $10046(3)$ & $42(1)$ \\
$\mathrm{C}(3)$ & $758(2)$ & $8307(2)$ & $8700(3)$ & $41(1)$ \\
$\mathrm{C}(4)$ & $752(2)$ & $8048(2)$ & $9773(4)$ & $50(1)$ \\
$\mathrm{C}(5)$ & $813(2)$ & $7508(2)$ & $9864(4)$ & $63(1)$ \\
$\mathrm{C}(6)$ & $887(2)$ & $7223(2)$ & $8905(5)$ & $70(1)$ \\
$\mathrm{C}(7)$ & $910(2)$ & $7481(2)$ & $7843(4)$ & $63(1)$ \\
$\mathrm{C}(8)$ & $849(2)$ & $8023(2)$ & $7714(3)$ & $45(1)$ \\
$\mathrm{C}(9)$ & $869(2)$ & $8273(2)$ & $6540(4)$ & $52(1)$ \\
$\mathrm{O}(1)$ & $890(2)$ & $7911(2)$ & $5713(3)$ & $86(1)$ \\
$\mathrm{O}(2)$ & $856(2)$ & $8757(1)$ & $6345(2)$ & $62(1)$ \\
$\mathrm{S}(2)$ & $919(1)$ & $8750(1)$ & $3007(1)$ & $98(1)$ \\
$\mathrm{O}(3)$ & $714(2)$ & $8140(2)$ & $3639(3)$ & $96(1)$ \\
$\mathrm{C}(10)$ & $1675(3)$ & $9269(3)$ & $3531(7)$ & $130(3)$ \\
$\mathrm{C}(11)$ & $519(3)$ & $9112(3)$ & $3627(5)$ & $115(2)$ \\
\hline
\end{tabular}

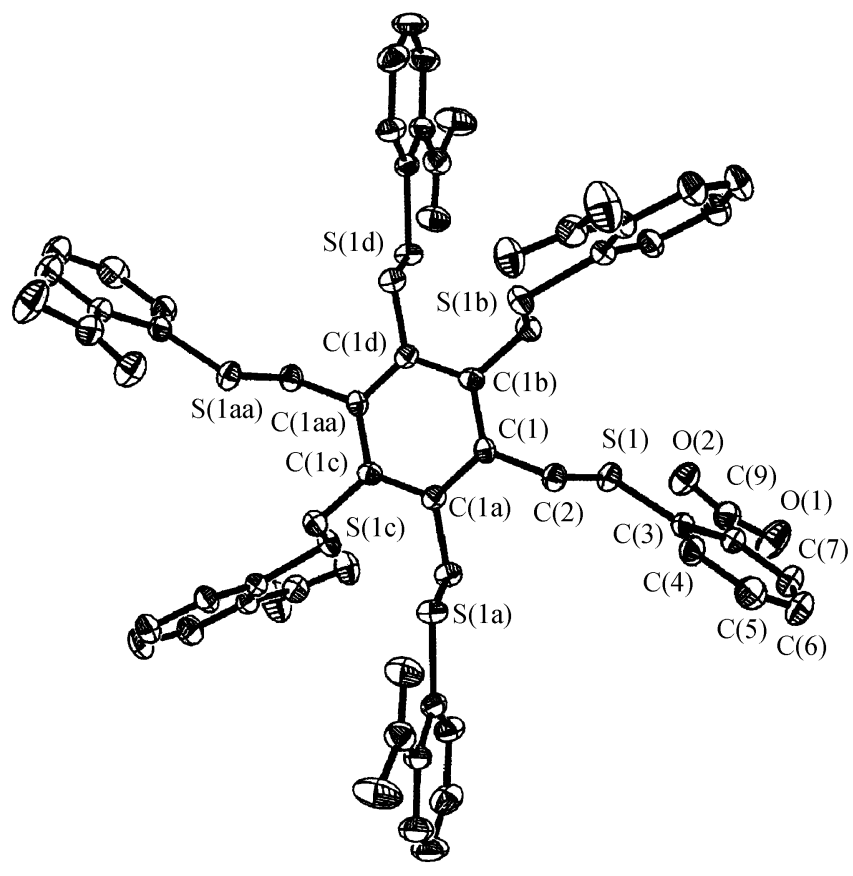

图 2 加合物 2 中 $1 \mathrm{H}_{6}$ 的分子结构图(ORTEP)

由于分子的热运动效应, 使得分子外围的键相对缩短, 这特别表现在端基苯环外侧的 $\mathrm{C}(5)-\mathrm{C}(6)$ 和 $\mathrm{C}(6)-\mathrm{C}(7)$ 键上(表 2). 与中心苯环相连的碳原子 $(\mathrm{C}(2), \mathrm{C}(2 \mathrm{a}), \cdots, \mathrm{C} 2(\mathrm{e})$ )离中心苯环 平面的距离小于 $0.008 \mathrm{~nm}$. 与端基苯环相连的碳原子 $(\mathrm{C}(9), \mathrm{C}(9 \mathrm{a}), \cdots, \mathrm{C} 9(\mathrm{e}))$ 与端基苯环平面 的距离小于 $0.01 \mathrm{~nm}$. 羧基氧原子 $(\mathrm{O}(1), \mathrm{O}(2) ; \cdots)$ 与端基苯环平面的距离不大于 $0.020 \mathrm{~nm}$. 六 个硫原子基本上位于端基苯环所在平面上(最大偏差 $0.013 \mathrm{~nm}$ ), 羧基 $\mathrm{COOH}$ 平面与相连的端 基苯环平面间夹角小于 $5.4^{\circ}$. 三角棱状 DMSO 的氧原子位于外侧, 两个甲基碳原子位于中心 
苯环平面的内侧, 朝向羧基内侧的酰基氧原子. 这种排列的结果是, 在中心苯环平面上下各形 成一个小口径的笼子, 类似中国传统的小口花瓶. 不过, 我们这里报道的这个“花瓶”是空瓶, 里面并没有包装客体分子. 根据这个花瓶的内腔尺寸, 容纳 DMSO, DMF 这类有机小分子是合 适的.

\section{3 铽配合物 3 的发光性能}

制备了星状六(苯甲酸)的系列稀土配合物, 发现铽配合物 $\mathbf{3}$ 发射明亮的绿色荧光(图 3), 而 铕配合物苂光很弱. 说明 1 能有效的敏化铽离子发光. 从吸收光谱看, 1 的电子跃迁主要定域 在端基苯甲酸上, 其紫外吸收谱与苯甲酸类似. 铽配合物 3 的固体粉末菼光光谱见图 3. 在紫 外区激发星状六苯甲酸的端基 $\pi-\pi$ 吸收带, 得到铽离子的特征苂光发射 ${ }^{5} \mathrm{D}_{4} \rightarrow{ }^{7} \mathrm{~F}_{\mathrm{J}}(J=6,5,4,3)$. 这里, 配体 1 作为典型的天线聚光色团, 较好地敏化了中心铽离子的苂光发射. 为了比较星状 六苯甲酸在敏化稀土发光方面的结构上的优势，我们在同样的条件下，即在热吡啶中制备了 苯甲酸琙配合物, 在同样的条件下测定了其苂光光谱(见图 3). 从图 3 可以看出, 3 的固体粉末 发光强度是相应苯甲酸铽配合物的 1.8 倍. 这种苂光增强效应不能单纯用配合物中存在的吡啶 来解释, 我们认为主要归于 1 对铽离子的有效包络作用.

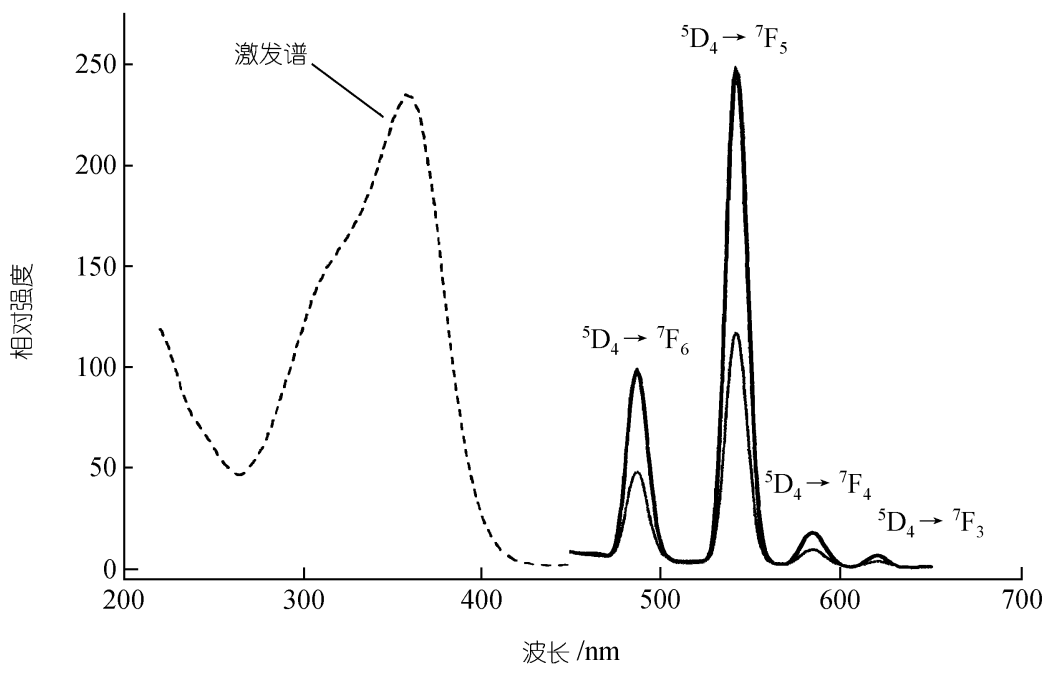

图 3 铽配合物 $\mathbf{3}$ 的室温固体粉末苂光光谱图

左边虚线: 激发光谱, 右边实线: 发射光谱. 细线是苯甲酸铽的发射光谱

在稀土有机配合物敏化发光的设计理念中，一条有效的途径既是利用穴状(Cryptand)或三 棱状(Tripodal)配体来稳定包络稀土离子, 屏蔽环境介质对稀土离子激发态的淬灭, 从而有效 的增强中心稀土离子的发光效率 ${ }^{[16]}$. 本文设计合成的六臂星状配体 $\mathbf{1}$, 能较有效的包络中心稀 土离子, 屏蔽了溶剂分子与金属离子的作用, 从而较相应的单分子苯甲酸能更好地敏化中心 铽离子的发光. 3 与相应苯甲酸铽的激发态 $\left({ }^{5} \mathrm{D}_{4}\right)$ 寿命分别为 1.73 和 $1.21 \mathrm{~ms}$, 与此结论吻合.

\section{参考文献}


2 Hardy A D U, MacNicol D D, Wilson D R. A new approach for the design of inclusion compounds. J Chem Soc, Perkin Trans II , 1979: $1011 \sim 1019$

3 Bourne S A, Nash K L G, Toda F. Inclusion compounds with a sexipedal host. Crystal structures and thermal analysis of inclusion compounds of hexakis(3-hydroxy-3,3-diphenylprop-2-ynyl)benzene with methyl ethyl ketone, diethyl ketone and dientyl ether. J Chem Soc, Perkin Trans II , 1996: 2145

4 Foces-Foces C, Llamas-Saiz A L, Claramunt R M, et al. Aromatic propellenes. Part 1. NMR spectroscopy, X-ray crystal and molecular structure of hexa(3,5-dimethypyrazol-1-yl)benzene. J Chem Soc, Perkin Trans II , 1995: 1359

5 Christensen C A, Bryce M R, Batsanov A S, et al. A novel hexakis(tetrathiafulvalene) derivative: Synthesis, structure and electrochemical properties. Chem Commun, 2000, $331 \sim 332$

6 Weiss R, Pomerehn B, Hampel F. First-ever per(onio) substitution of benzene: The role of the counterion. Angew Chem Int Ed Engl, 1995, 34: 1319 1321

7 Lambert C, Nöll G. Optically and Thermally Induced Electron Transfer Pathways in Hexakis[4-(N,N-diarylamino)phenyl] benzene Derivatives. Chem Eur J, 2002, 8: 3467 3477

8 Yang C, Chen X M, Yang Y S. First metal-encapsulating star polysulfoxide incorporating 2-amino-1, 3, 4-thiadiazole. Chem Commun, 1997: $2041 \sim 2042$

9 Kobayashi K, Shirasaka T, Sato A, et al. Self-assembly of a radially functionalized hexagonal molecule: hexakis (4hydroxyphenyl) benzene. Angew Chem Int Ed, 1999, 38, $3483 \sim 3486$

10 Kobayashi K, Shirasaka T, Horn E, et al. Two-dimensional hexagonal hydrogen-bonded network with triangle-like large cavites: Hexakis(4-carboxyphenyl)benzene. Tetra Lett, 2000, 41: 89 93

11 Elwahy A H M. A new approach for the design of novel hexa-host molecules. Tetra Lett, 2001, 42: $5123 \sim 5126$

12 Hoskins B F, Robson R, Slizys D A. A hexaimidazole ligand binding six octahedral metal ions to give an infinite 3D a-Po-like network through which two independent 2D hydrogen-bonded networks interweave. Angew Chem Int Ed Engl, 1997, 36: $2752 \sim 2755$

13 Dijkstra H P, Steenwinkel P, Grove D M, et al. Design of novel hexametallic cartwheel molecules from persubstituted benzene compounds. Angew Chem Int Ed, 1999, 38: $2185 \sim 2188$

14 Yip H K, Schier A, Riede J,et al. J Chem Soc, Dalton Trans, 1994: 2333

15 Sheldrich G M. SHELXL-93, Program for crystal structure refinement, University of Göttingen, 1993

16 杨 迟, 杨燕生. 发光镧系超分子的设计及应用. 《今日化学》《大学化学》编辑部编. 北京: 北京大学出版社, 1995. 94 98 